A Final Technical Report

on

\title{
Configurational Forces in Solid Nanostructures
}

\author{
DE-FG02-99ER45787 \\ September 1999 to June 2003 \\ by \\ Zhigang Suo \\ Mechanical and Aerospace Engineering Department \\ and Princeton Materials Institute \\ Princeton University \\ Princeton, NJ 08544 \\ 609-258-0250, Suo@Princeton.EDU, FAX 609-258-5877 \\ Submitted to \\ Dr. Yok Chen \\ U. S. Department of Energy \\ SC-131 Germantown Building \\ Division of Material Science \\ 1000 Independence Avenue, SW \\ Washington D. C. 20585-1290 \\ Phone: (301) 903-4174 \\ FAX: (301) 903-9513 \\ Email: Yok.Chen@Science.doe.gov
}

June 2003 


\section{SUMMARY}

The DOE grant (DE-FG02-99ER45787) to Princeton University, entitled Configurational Forces in Solid Nanostructures, was intended to cover the four-year period from September 1999 to September 2003. Effective 1 July 2003, the PI will relocate from Princeton to join the Harvard faculty. Princeton University will submit the Final Financial Report, the Final Property Report, and the Final Patent Report. The expenditures to date are \$261,513 with \%8,487 remaining of the awarded amount of $\$ 320,000$. Harvard University will submit a request for the remaining amount. This Final Technical Report covers from the period between September 1999 to June 2003.

Three Ph.D. students, Wei Lu, Yanfei Gao and Wei Hong, admitted to Princeton in the fall of 1998, 1999, 2002, respectively, have been dedicated to this project. Wei Lu earned his Ph.D. in August 2001, and is now an assistant professor at The University of Michigan, Ann Arbor. Yanfei Gao earned his Ph.D. in February 2003, and is now a post-doc at Brown University. The amount of funding covers one student at a time. All three students received first-year fellowships from Princeton University. In the Mechanical and Aerospace Engineering Department, to fulfill a doctoral degree requirement, every student serves as a teaching assistant for three semesters, for which the student is partially paid by the University. The project has resulted in 12 publications [1-12].

\section{SCEINTIFIC ACHIEVEMENTS}

For more than a decade, experimental evidence has accumulated that a monolayer of atoms or molecules, adsorbed on a solid surface, can self-assemble into various phase patterns, including periodic stripes, triangular lattices, square lattices, and irregular arrangements [13-20]. The feature size of the patterns is on the order of 1-100 nm, and is often stable on annealing.

As demonstrated by Whitesides and his co-workers, contact-printed micron-scale monolayer patterns can be used either as resists in selective wet etching, or as templates in selective deposition [21]. Similar uses of the self-assembled nanoscale monolayer patterns have been attempted to fabricate functional structures [22].

Phase Refining

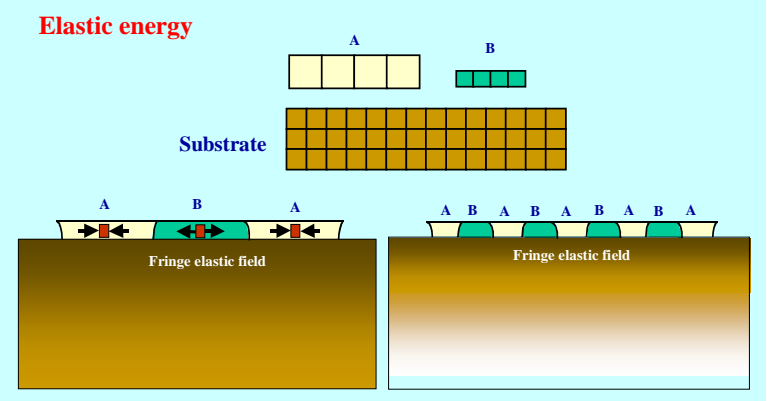

Fig. 1

Tantalizing technological potentials aside, this project has focused on the theoretical challenges raised by the experimental observations of self-assembled monolayer patterns. The patterns form to minimize the combined free energy of mixing, phase boundary, and elastic field. Fig. 1 illustrates the main idea using an epitaxial film grown on a substrate [2]. Say the film forms two domains, A and B. Domain A has a larger lattice constant than the substrate and would be under compression on the substrate. Domain B has a smaller lattice constant than the substrate and would be under tension on the substrate. When they alternate on the substrate, the two kinds of domains compensate: the elastic energy of the polydomain film on the substrate is lower than a monodomain film on the substrate. The mismatch in the polydomain still causes a fringe elastic field in the substrate, whose depth scales with the domain size. When the domain size is refined, the fringe field depth is reduced, and so 
is the elastic energy. Consequently, the elastic energy in the film-substrate composite tends to refine the domains. The refinement, however, adds more domain wall areas, which increases the total domain wall energy. That is, the domain wall energy tends to coarsen the domains. The elastic energy is mediated by a long-range field. The domain wall energy is mediated by shortrange interactions among atoms at the domain wall. The two competing actions-refining and coarsening — can select an equilibrium domain size.

The model of Vanderbilt and co-workers [23,24] highlights the competition between the phase boundary energy and the elastic energy in selecting the phase size. The model assumes that the phase boundary is a mathematical line endowed with an excess energy per unit length. Certain phase patterns, such as periodic stripes and triangular lattices are assumed, and the feature size is determined by minimizing the free energy.

Three difficulties arise in such a model. First, when the phase boundary is represented as a mathematical line, the surface stress jumps across the phase boundary, leading to a singular elastic field. The elastic energy is unbounded, and the prediction of the equilibrium feature size depends on how the problem is regularized. Second, because the phase patterns are assumed, the true energy minimizer may lie outside the "pool of the candidates". Third, experimental observations have shown that perfect lattice is an exception, rather than the rule. Various imperfections are the consequence of the kinetic process. An energetic model is inadequate.

We have developed a continuous phase field model of the Cahn-Hilliard type [1]. The model represents the phases by a continuous concentration field, and the phase boundaries by concentration gradients. Consequently, our model may be regarded as a specific way to regularize the problem. For a given concentration field, the surface stress is nonuniform but continuous, inducing an elastic field in the substrate. The concentration field of the monolayer and the elastic field of the substrate co-evolve. The model does not presume the pattern type; rather, starting from an initial concentration field, a diffusion equation evolves the concentration field, leading to the pattern. Our simulations have revealed a great variety of imperfect structures, and several surprises.

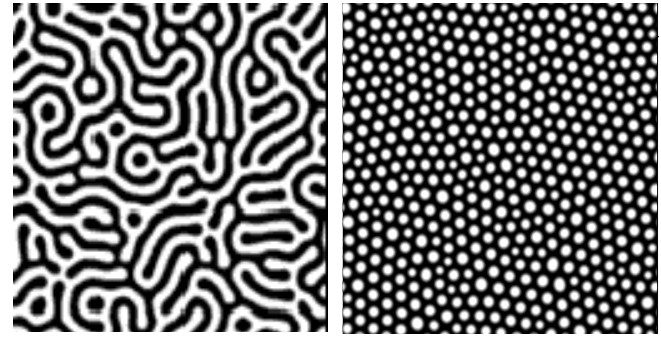

(a)

(b)
Fig. 2 (a) The concentration field initially fluctuates randomly around the average concentration $C_{a v}=0.5$, and evolves into a pattern of meandering stripes. (b) The concentration field initially fluctuates randomly around the average concentration $C_{a v}=0.4$, and evolves into a pattern of dots. The patterns shown here have remained stable for a long time.

Fig. 2 shows simulation results for two average concentrations, $C_{a v}=0.5$ and $C_{a v}=0.4$ [4]. In both cases, the initial concentration fields randomly fluctuate around the averages. Fig. 2a shows the concentration field after the annealing time $t=10^{5} \tau$, where $\tau$ is the time scale in the model. At around $t=10^{2} \tau$, the monolayer already separates into two phases of meandering stripes. The pattern and the feature size hardly change between $10^{2} \tau$ to $10^{5} \tau$. Fig. $\mathbf{2 b}$ shows a pattern at time $t=4 \times 10^{6} \tau$. The dots are established around $t=10^{2} \tau$. Further annealing does not change the size of the dots appreciably, but improves the spatial ordering of the dots somewhat. At $t=4 \times 10^{6} \tau$ shown in Fig. $2 \mathbf{b}$, the pattern consists of grains, each grain being a triangular lattice consisting of fewer than ten dots across. 
These simulations clearly show the effect of symmetry on pattern formation. The model used for the simulation is isotropic, with no preferred orientation in the plane of the monolayer. Consequently, stripes of all orientations are equally possible, so are lattices of disks of all orientations. Improving the long-range order by annealing alone takes a long time. One can remove the confusion by breaking the symmetry. Fig. 3 shows an evolution sequence at six times [4]. On top of the same random initial concentration field we add three lines having concentration $C=0.51$. The three lines seed the superlattice, inducing stripe colonies by consuming the nearby serpentine structures. At $t=100$, two colonies meet, producing a pair of dislocations. Each dislocation moves by climbing. Periodic stripes form in the entire calculation cell within $t=10^{5}$. The present simulation suggests that serpentine structures can transform into an array of stripes if one breaks the symmetry at a coarse scale.

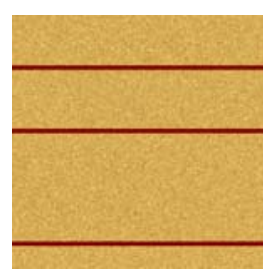

$\mathrm{t}=0$

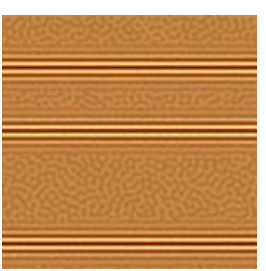

$\mathrm{t}=10$

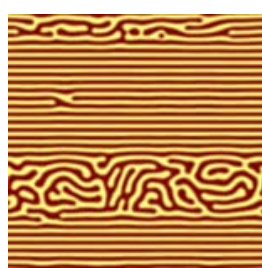

$\mathrm{t}=100$

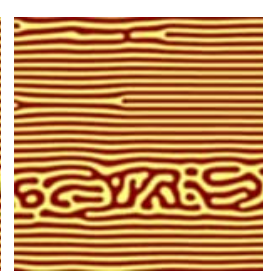

$\mathrm{t}=500$

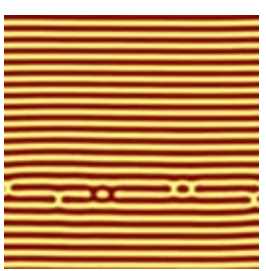

$\mathrm{t}=10000$

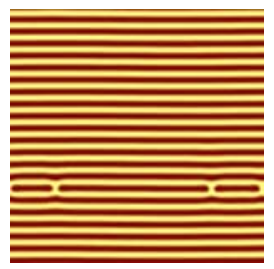

$\mathrm{t}=40000$

Fig. 3 Simulation starts with a random initial concentration field plus three lines with $C=0.51$. The average concentration equals 0.5 .

To describe the renewal project in concrete terms in later sections, here we outline our existing theory [6]. Let $x_{1}, x_{2}, x_{3}$ be the coordinates, and the substrate surface coincide with the plane $\left(x_{1}, x_{2}\right)$. Although the monolayer pattern is two-dimensional, the elastic field in the substrate is three-dimensional. Let $C\left(x_{1}, x_{2}\right)$ be the concentration field of the monolayer, and $u_{i}\left(x_{1}, x_{2}, x_{3}\right)$ be the displacement field in the substrate. The strain tensor, $\varepsilon_{i j}$, relates to the displacement vector, $u_{i}$, as

$$
\varepsilon_{i j}=\frac{1}{2}\left(u_{i, j}+u_{j, i}\right) .
$$

A Latin subscript runs from 1 to 3.

Split the free energy $G$ of the monolayer-substrate system into two parts. The elastic field in the system gives the volumetric energy. Define the surface energy as the free energy of the system in excess of the volumetric energy. The free energy functional takes the form

$$
G=\int W d V+\int \Gamma d A .
$$

The elastic energy per unit volume, $W$, is integrated over the volume of the substrate. The excess surface energy per unit area, $\Gamma$, is integrated over the surface area covered by the monolayer.

The substrate is elastic and anisotropic, with the stiffness tensor $c_{i j k l}$. The elastic energy density is quadratic in the strain:

$$
W=\frac{1}{2} c_{i j k l} \varepsilon_{i j} \varepsilon_{k l} .
$$

Repeated subscripts imply summation.

The surface energy density $\Gamma$ is a function of the concentration $C$, the concentration gradient $C_{, \alpha}$, and the strains in the surface, $\varepsilon_{\alpha \beta}$. Greek subscripts run from 1 to 2 . Expand the 
surface energy density to the leading order power series in the concentration gradient and the strain, and we have

$$
\Gamma=g+h C_{, \beta} C_{, \beta}+f_{\alpha \beta} \varepsilon_{\alpha \beta} .
$$

The leading term in the concentration gradient is quadratic, because the symmetry excludes the term linear in the concentration gradient.

When the concentration field is uniform, the surface stress is also uniform, so that the semi-infinite substrate is unstrained, and only $g$ remains in (4). Consequently, $g$ represents the free energy of mixing, and is a function of the concentration. In simulation, we have assumed that the monolayer forms a binary regular solution, so that

$$
g(C)=\Lambda k T[C \ln C+(1-C) \ln (1-C)+\Omega C(1-C)] .
$$

Here $\Lambda$ is the number of surface sites per unit area. The dimensionless parameter $\Omega$ measures the magnitude of the enthalpy of mixing relative to that of the thermal energy. When $\Omega<2$, the function $g(C)$ is convex, and the monolayer forms a solution. When $\Omega>2$, the function $g(C)$ has two wells, and the monolayer separates into two phases.

The phase boundary energy is represented by the term quadratic in the concentration gradient in Equation (4). The coefficient $h$ is in general a function of the concentration and the direction of the concentration gradient. The phase boundary energy can be calculated from an atomistic model, but we are unaware of any such calculations for the two-phase monolayers. Here we will simply treat the function $h$ as a phenomenological constant. We have also introduced anisotropy of $h$.

Assume that the surface stress tensor $f_{\alpha \beta}$ is linear in the concentration $C$. That is, when the concentration changes by $\Delta C$, the surface stress changes by $\Delta f_{\alpha \beta}=\phi_{\alpha \beta} \Delta C$. The slope tensor $\phi_{\alpha \beta}$ depends on the materials system, and can be measured by the wafer curvature method [25].

The system varies its free energy by two means: the elastic deformation in the substrate, and atomic diffusion in the monolayer. Given a displacement field in the substrate, $u_{i}\left(x_{1}, x_{2}, x_{3}\right)$, and the concentration field in the monolayer, $C\left(x_{1}, x_{2}\right)$, we can calculate the free energy. The system strives to attain both elastic and diffusive equilibria. The system attains elastic equilibrium when the free energy variation associated with the variation in the displacement field vanishes. This leads to an elasticity boundary value problem for the substrate, with the traction vector on the surface of the substrate prescribed as

$$
\sigma_{3 \alpha}=\phi_{\alpha \beta} C_{, \alpha}, \sigma_{33}=0
$$

For a given concentration field $C\left(x_{1}, x_{2}\right)$, the elastic field in the substrate is determined by solving the boundary value problem.

The system reaches diffusive equilibrium when the free energy variation with the concentration field vanishes. The equilibrium phase pattern must attain both elastic and diffusive equilibria. Elastic relaxation is much faster than surface diffusion, so that we assume that the system maintains the elastic equilibrium at all time. For a given concentration field, the equilibrium elastic field is determined by the boundary value problem described above. When the concentration field changes, the change in the free energy define a thermodynamic force, which in turn drives the concentration field change. We have derived a diffusion equation to evolve the concentration field:

$$
\frac{\partial C}{\partial t}=\frac{M}{\Lambda^{2}} \nabla^{2}\left(\frac{\partial g}{\partial C}-2 h \nabla^{2} C+\phi_{\alpha \beta} \varepsilon_{\alpha \beta}\right),
$$


where $M$ is the mobility of the molecules on the surface. In the parentheses are forces that drive the self-assembly. The first term comes from the free energy of mixing, and drives phase separation. The second term comes from the phase boundary energy, and drives phase coarsening. The third term comes from the elastic energy, and drives phase refining. It is the third term that couples the two-dimensional concentration field of the monolayer to the three dimensional elastic field in the substrate.

We have solved the three-dimensional, anisotropic elasticity boundary value problem analytically in the reciprocal space $[8,10]$. Because the function $g(C)$ is nonlinear, we have adopted a numerical method that repeatedly goes between the real and reciprocal spaces by using the fast Fourier transform (FFT) [4].

\section{IMPACT AND FUNTURE OUTLOOK}

Mass-producing functional structures at the nanoscale is a great challenge of our time. Various self-assembly processes offer tantalizing promises as low cost, high throughput patterning techniques. However, both recent experimental observations and our own theoretical simulations have shown that self-assembly processes seldom produce perfect periodic structures, and never produce designed aperiodic structures. On the other hand, nature has produced proteins, polypeptides of specific, aperiodic sequences. The lesson, learned from our own frustration and from nature's solution, is to template the self-assembly. The proposed project builds on our accomplishments in modeling self-assembled monolayer patterns, and on our strength in solid mechanics, in which a large collection of tools are available to solve complex boundary value problems. To make definitive progress, our research focuses on a class of selfassembling systems (the monolayer on solid substrate). These systems exhibit rich phenomena, and are model systems to study a wide range of issues inherent in self-assembly. It is envisioned that our theory will not only explain the available experimental observations, but also motivate new experiments, especially in using various template mechanisms and unconventional substrates. The field is wide open.

\section{REFERENCES}

PDF files of reference 1-12 are available on line, www.princeton.edu/ suo

1. Z. Suo and W. Lu, Composition modulation and nanophase separation in a binary epilayer. Journal of the Mechanics and Physics of Solids, 48, 211-232 (2000).

2. W. Lu and Z. Suo, Coarsening, refining, and pattern emergence in binary epilayers. Zeitschrift fur Metallkunde, 90, 956-960 (1999).

3. Z. Suo and W. Lu, Self-organizing nanophases on a solid surface, pp. 107-122, in MultiScale Deformation and Fracture in Materials and Structures-The James R. Rice $60^{\text {th }}$ Anniversary Volume edited by T.-J. Chuang and J. W. Rudnicki, KLUWER ACADEMIC PUBLISHERS, The Netherlands (2000).

4. W. Lu and Z. Suo, Dynamics of nanoscale pattern formation of an epitaxial monolayer. Journal of the Mechanics and Physics of Solids, 49, 1937-1950 (2001).

5. Y. Gao, Z. Suo Domain dynamics in a ferroelastic epilayer on a paraelastic substrate. Journal of Applied Mechanics, 69, 419-424 (2002).

6. Z. Suo and W. Lu, Forces that drive self-assembly on solid surfaces. Journal of Nanoparticles Research, 2, 333-344 (2000). 
7. W. Lu and Z. Suo, Symmetry breaking in self-assembled monolayers on solid surfaces. I. Anisotropic surface stress. Phys. Rev. B 65, 085401 (2002).

8. W. Lu and Z. Suo, Symmetry breaking in self-assembled monolayers on solid surfaces. II. Anisotropic substrate elasticity. Phys. Rev. B 65, 205418 (2002).

9. Y.F. Gao, W. Lu and Z. Suo, A mesophase transition in a binary monolayer on a solid surface. Acta Materialia, 50, 2297-2308 (2002).

10. Y.F. Gao and Z. Suo, The orientation of the self-assembled monolayer stripes on a crystalline substrate. Journal of The Mechanics and Physics of Solids. 51, 147-167 (2003).

11. Z. Suo, Y.F. Gao and G. Scoles, Nanoscale domain stability in organic monolayers on metals. Submitted to Journal of Applied Mechanics. August 2002.

12. Y.F. Gao and Z. Suo, Guided self-assembly of molecular dipoles on a substrate surface. Journal of Applied Physics. Schedule to appear in the issue of April 1, 2003

13. Kern, K., Niehus, H., Schatz, A., Zeppenfeld, P., Goerge, J., Comsa, G., 1991. Long-range spatial self-organization in the adsorbate-induced restructuring of surfaces: $\mathrm{Cu}\{110\}$ $(2 \times 1)$ O. Phys. Rev. Lett. 67, 855-858.

14. Leibsle, F.M., Flipse, C.F.J., Robinson, A.W., 1993. Structure of the $\mathrm{Cu}\{100\}-\mathrm{c}(2 \times 2) \mathrm{N}$ surface: a scanning-tunneling-microscopy study. Phys. Rev. B 47, 15865-15868.

15. Zeppenfeld, P., Krzyzowski, M.A., Romainczyk, Ch., Comsa, G., Lagally, M.G., 1994. Size relation for surface systems with long-range interactions. Phys. Rev. Lett. 72, 2737-2740.

16. Zeppenfeld, P., Krzyzowski, M.A., Romainczyk, Ch., David, R., Comsa, G., Röder, H., Bromann, K., Brune, H., Kern, K., 1995. Stability of disk and stripe patterns of nanostructures at surfaces. Surf. Sci. 342, L1131-L1136.

17. Pohl, K., Bartelt, M.C., de la Figuera, J., Bartelt, N.C., Hrbek, J., Hwang, R.Q., 1999. Identifying the forces responsible for self-organization of nanostructures at crystal surfaces. Nature 397, 238-240 (1999).

18. Ellmer, H., Repain, V., Rousset, S., Croset, B., Sotto, M., Zeppenfeld, P., 2001. Self-ordering in two dimensions: nitrogen adsorption on copper (100) followed by STM at elevated temperature. Surf. Sci. 476, 95-106.

19. Plass, R., Last, J.A., Bartelt, N.C., Kellogg, G.L., 2001. Self-assembled domain patterns. Nature 412, 875-875.

20. Plass, R., Bartelt, N.C., Kellogg, G.L., 2002. Dynamic observations of nanoscale selfassembly on solid surfaces. Journal of Physcs: Condensed Matter 14, 4227-4240 (2002).

21. Xia, Y., Rogers, J.A., Paul, K.E., Whitesides, G.M., 1999, Unconventional methods for fabricating and patterning nanostructures. Chem. Rev. 99, 1823-1848.

22. Li, D., Diercks, V., Pearson, J., Jiang, J.S., Bader, S.D., 1999. Structural and magnetic studies of fcc Fe films with self-organized lateral modulation on striped $\mathrm{Cu}(110)-\mathrm{O}(2 \times 1)$ substrates. J. Appl. Phys. 85, 5285-5287.

23. Alerhand, O.L., Vanderbilt, D., Meade, R.D., Joannopoulos, J.D., 1988. Spontaneous formation of stress domains on crystal surfaces. Phy. Rev. Lett. 61, 1973-1976.

24. Ng, K.-O., Vanderbilt, D., 1995. Stability of periodic domain structures in a two-dimensional dipolar model. Phys. Rev. B 52, 2177-2183.

25. Ibach, H., 1997. The role of surface stress in reconstruction, epitaxial growth and stabilization of mesoscopic structures. Surf. Sci. Rep. 29, 195-263. Erratum. Surf. Sci. Rep. 35, 71-73. 